\section{BMJ Paediatrics Open}

\title{
Efficacy and safety of vasopressin and terlipressin in preterm neonates: a protocol for a systematic review
}

To cite: Alsaadi AS, Sushko K Bui V, et al. Efficacy and safety of vasopressin and terlipressin in preterm neonates: a protocol for a systematic review. BMJ Paediatrics Open 2021;5:e001067. doi:10.1136/ bmjpo-2021-001067

- Additional supplemental material is published online only. To view, please visit the journal online (http://dx.doi.org/ 10.1136/bmjpo-2021-001067).

Received 16 February 2021 Accepted 13 May 2021

\section{Check for updates}

\section{Author(s) (or their} employer(s)) 2021. Re-use permitted under CC BY-NC. No commercial re-use. See rights and permissions. Published by BMJ.

For numbered affiliations see end of article.

Correspondence to Dr Abdulrahman Salim Alsaadi; dr-abdul-alsaadi@hotmail.com

\section{ABSTRACT}

Background The use of vasoactive agents like arginine vasopressin (AVP) and terlipressin to treat hypotension or persistent pulmonary hypertension in critically ill preterm neonates is increasing. Therefore, a systematic review of the available data on dosing, efficacy and safety of AVP and terlipressin in this patient population appears beneficial. Methods We will conduct a systematic review of the available evidence on the use of AVP and terlipressin for the treatment of hypotension or persistent pulmonary hypertension in preterm neonates. We will search Ovid MEDLINE, EMBASE, the Cochrane Central Register of Controlled Trials, Web of Science and Google Scholar from inception to March 2021. Two reviewers will independently screen titles and abstracts, review the full text of eligible studies, extract data, assess the risk of bias and judge the certainty of the evidence. Our primary outcome will be an (1) improvement of end-organ perfusion after initiation of AVP or terlipressin and (2) mortality prior to discharge. Our secondary outcomes will include (1) major neurosensory abnormality and (2) the occurrence of adverse events. Discussion The currently available evidence on the efficacy and safety of AVP and terlipressin in preterm neonates is limited. Yet, evidence on the pharmacology of these drugs and the pathophysiology of vasoplegic shock support the biological plausibility for their clinical effectiveness in this population. Therefore, we aim to address this gap concerning the use of vasopressin and terlipressin among critically ill preterm neonates.

Trial registration This protocol has been submitted for registration to the international database of prospectively registered systematic reviews (PROSPERO, awaiting registration number).

\section{INTRODUCTION}

Arginine vasopressin (AVP) is a potent endogenous peptide hormone that regulates extracellular volume and renal water excretion via vasopressin receptor 2 (V2R). ${ }^{1}$ At supraphysiologic concentrations, AVP exerts moderate vasoconstrictor effects via vasopressin receptor 1 (V1R). ${ }^{2}$ The contradictory actions of vasopressin, however, appear to be mainly related to the distribution pattern and function of receptors (vasoconstriction via V1R in vascular smooth muscle and vasodilation via

\section{What is already known on this topic?}

Arginine vasopressin (AVP) and terlipressin are potent systemic vasoconstrictors and pulmonary vasodilators.

- The efficacy of AVP and terlipressin to treat septic shock and haemodynamic instability in adult and paediatric patients is well established.

- There is a lack of data on how to treat refractory shock, a leading cause of morbidity and mortality, in preterm neonates.

\section{What this study hopes to add?}

Data on the efficacy of AVP and terlipressin for the treatment of hypotension and persistent pulmonary hypertension in critically ill preterm neonates.

- Data on the safety of AVP and terlipressin for the treatment of hypotension and persistent pulmonary hypertension in critically ill preterm neonates.

- Data on important evidence gaps in need of future research for optimal use of AVP and terlipressin in critically ill neonates with hypotension and persistent pulmonary hypertension.

V2R expressed in endothelium by NO formation). ${ }^{3-6}$

The use of AVP to restore vascular tone in vasoplegic shock was described for the first time in $1997 .^{7}$ Since then, a number of studies have illustrated the efficacy of AVP in catecholamine-resistant shock in adults and children, with limited data available for the use of AVP to treat neonatal catecholamine-resistant septic, cardiogenic shock and refractory persistent pulmonary hypertension. ${ }^{8-10}$ In 2006, Meyer et al reported the first use of AVP in six extremely low birthweight infants with catecholaminerefractory shock and acute renal injury. ${ }^{10}$ Following the administration of AVP, investigators observed a substantial increase in arterial blood pressure and urine output among three infants with septic shock and a 
mortality rate of one out of three. However, among the infants with non-septic shock, blood pressure and urine output only improved briefly, and the mortality rate was three out of three. ${ }^{10}$

Similar to AVP, terlipressin, a synthetic long-acting analogue of vasopressin, has potent vasoconstrictor properties. Terlipressin, however, selectively binds to V1R and has a longer half-life and duration of action. Currently available data demonstrate that vasopressin has an elimination half-life of $\leq 10 \mathrm{~min}$ with onset of its pressor effect within $15 \mathrm{~min}$ that fades within $20 \mathrm{~min}$ after stopping the infusion. For terlipressin, the estimated half-life is up to 3 hours with time to peak plasma concentration of approximately 2 hours and duration of action of 4-6hours. ${ }^{11}$ Among adults and children with septic shock, terlipressin improves haemodynamic parameters and reduces the use of catecholamines. However, reports have also highlighted the controversial effects of terlipressin on survival, and also described tissue ischaemia as a possible adverse event. ${ }^{12-15}$

In premature neonates treated in neonatal intensive care units (NICUs), fluid-refractory catecholamineresistant shock remains a leading cause of morbidity and mortality. ${ }^{16}$ Hence, there is an immediate need for pharmacotherapeutic options that can target the intractable vasoplegia via alternative pathways. Evidence suggests that AVP and terlipressin have favourable haemodynamic profiles as they lead to a minimal decline in cardiac output, induce limited vasoconstriction in the pulmonary circulation and cause selective constriction of renal efferent over afferent arterioles. ${ }^{17-19}$ This data, along with reports of low levels of circulating AVP in patients with refractory shock, provide a biologic plausibility and support the use of these drugs by NICU clinicians. ${ }^{20}$ This has resulted in a considerable increase in the use of AVP and its analogues in preterm neonates. ${ }^{21}{ }^{22}$ Despite the promising outlook, the evidence on the efficacy and safety of AVP and its analogues remains controversial, particularly regarding the short-term and long-term adverse effects in preterm neonates. ${ }^{23-26}$ Reports of serious adverse events such as decreased cardiac index, peripheral cyanosis and digital ischaemia are among complications that require further investigation. ${ }^{26} \mathrm{~A}$ recent systematic review and meta-analysis that examined the role of vasopressin and terlipressin in refractory shock in paediatric patients, reported improvements in haemodynamic indices but lack of benefit on mortality or length of hospital stay with a trend towards a higher risk for tissue ischaemia. ${ }^{24}$ In critically ill preterm neonate with complex and poorly understood hormonal dysfunction, the risk of such undesired short-term and long-term adverse effects is of serious concern. ${ }^{2123}$ In view of the increasing use of AVP and terlipressin in neonates of NICUs and high risk of serious adverse events, the need for enhancement of data on efficacy and safety of these drugs in this vulnerable population becomes clear.

\section{Objective}

We aim to conduct a systematic review of the available evidence on the dosing, efficacy, and safety of AVP and terlipressin in preterm neonates with hypotension or persistent pulmonary hypertension (box 1 ).

\section{METHODS}

\section{Protocol registration}

The protocol for this systematic review has been submitted to the international database of prospectively registered systematic reviews (PROSPERO, awaiting registration number). The development and reporting of the protocol followed the Preferred Reporting Items for Systematic Review and Meta-Analysis Protocol (PRISMA-P) guidelines. ${ }^{27}$ The final review will also be reported according to the PRISMA guidelines.

\section{Data sources and search strategy}

We developed a search strategy in consultation with a professional librarian to comprehensively search Ovid MEDLINE (1964 to February 2021), EMBASE (1974 to March 2021), Web of Science (1900 to March 2021) and the Cochrane Central Register of Controlled Trials (CENTRAL) (online supplemental appendix A). This search strategy, containing database-specific subject headings and text word terms for concepts, was first developed in MEDLINE (Ovid interface) and was translated as appropriate for the other databases. We also plan to search the bibliographies of any relevant studies for additional references. Using Google Scholar, we will also seek out relevant studies that are not commercially published, such as conference abstracts, dissertations,

\section{Box 1 PICO framework}

Population: Preterm neonates born at less than 37 weeks' gestation with hypotension (defined as mean blood pressure less than gestational age or hypotension requiring fluid or vasoactive therapy) or persistent pulmonary hypertension.

Intervention: Arginine vasopressin or terlipressin administered intravenously, initiated at any time and for any duration as a primary or rescue treatment for hypotension or persistent pulmonary hypertension.

Comparator: Standard treatment, placebo or any other vasoactive agent.

Outcomes: (1) Improvement in end-organ perfusion defined as an increase in mean, diastolic or systolic blood pressure within 1 hour of the start of treatment, or an increase in urine output, a decrease in the need for inotropes, or a reduction in serum lactate within 12 hours of treatment initiation as identified in the primary studies, (2) mortality prior to discharge. Our secondary outcomes (1) major neurosensory disability defined as moderate to severe motor or cognitive impairment or severe visual or hearing impairment as identified in the primary study; and (2) the occurrence of adverse events defined as reports of peripheral tissue ischaemia, gastrointestinal events (occurrence of perforation, necrotising enterocolitis or gastrointestinal bleed), hepatic events, renal events or hyponatraemia as identified in the primary studies. (table 2 ). 


\begin{tabular}{ll}
\hline Table 1 Data extraction form for primary studies \\
\hline Study ID & \\
\hline Title & \\
\hline Author & USA \\
Country of study conduct & Canada \\
& Australia \\
& Other \\
& \\
\hline Study characteristics & \\
\hline Aim & Randomised controlled trial \\
Design & Non-randomised experimental \\
& study \\
& Cohort study \\
& Cross-sectional study \\
& Case-control study \\
& Case series \\
& Case report \\
& Other \\
\hline
\end{tabular}

\begin{tabular}{|c|c|}
\hline Start date & \\
\hline End date & \\
\hline Funding source & \\
\hline Conflict of interest & \\
\hline Participant characteristics & \\
\hline Population description & \\
\hline Inclusion criteria & \\
\hline Exclusion criteria & \\
\hline Sample size (n) & \\
\hline Birth weight (grams) & \\
\hline Gestational age (weeks) & \\
\hline Postnatal age (weeks) & \\
\hline Underlying condition & \\
\hline Comorbidities & \\
\hline Concurrent medications & \\
\hline Intervention/exposure details & \\
\hline $\begin{array}{l}\text { AVP or terlipressin dosing (mg/ } \\
\mathrm{kg} / \text { dose) }\end{array}$ & \\
\hline $\begin{array}{l}\text { AVP or terlipressin treatment } \\
\text { duration (days) }\end{array}$ & \\
\hline $\begin{array}{l}\text { AVP or terlipressin route of } \\
\text { administration }\end{array}$ & \\
\hline Control details & \\
\hline Pharmacotherapy & $\begin{array}{l}\text { Dopamine } \\
\text { Dobutamine } \\
\text { Epinephrine } \\
\text { Norepinephrine } \\
\text { Placebo } \\
\text { Other }\end{array}$ \\
\hline Outcomes & \\
\hline Primary outcomes & \\
\hline $\begin{array}{l}\text { Improvement in end organ } \\
\text { perfusion details }\end{array}$ & $\begin{array}{l}\text { SBP } \\
\text { DBP } \\
\text { MBP } \\
\text { Urine output } \\
\text { Inotropic support } \\
\text { Serum lactate }\end{array}$ \\
\hline Mortality & Death before discharge \\
\hline
\end{tabular}

Continued

\begin{tabular}{ll}
\hline Table 1 Continued & \\
\hline Study ID & \\
\hline Secondary outcomes $^{*}$ & \\
\hline Major neurosensory disability & Neurosensory disability \\
& $\begin{array}{l}\text { Moderate to severe motor or } \\
\text { cognitive impairment } \\
\text { Severe visual or hearing } \\
\text { impairment }\end{array}$ \\
& Adverse events \\
Occurrence of adverse events & Peripheral tissue ischaemia \\
& Gastrointestinal events \\
& Renal events \\
& Hepatic events \\
& Hyponatraemia
\end{tabular}

${ }^{*}$ The secondary outcomes will be defined as reported in the primary studies. AVP, arginine vasopressin; DBP, diastolic blood pressure; MBP, mean blood pressure; SBP, systolic blood pressure.

policy documents and book chapters. We will not apply any language or study design limitations. Animal studies and duplicate studies will be excluded. A peer-review of our strategy using the Peer Review for Electronic Search Strategies guideline was completed by a professional librarian $^{28}$ (online supplemental appendix B).

\section{Eligibility criteria}

All interventional and observational original research studies, including randomised controlled trials (RCTs), quasi RCTs, prospective and retrospective cohort studies, descriptive studies, case series and case reports and conference abstracts describing the use of AVP or terlipressin as primary or rescue treatment for hypotension or persistent pulmonary hypotension in hospitalised preterm neonates born at less than 37 weeks' gestation will be eligible for inclusion. We will include studies with mixed populations (term and preterm neonates) as long as separate data for preterm neonates are available. Studies will be included irrespective of the dose, administration frequency and duration of AVP or terlipressin treatment. In studies with a control group, the provided intervention(s), placebo or standard practice will be the comparator. In studies with no comparator group, the observational report of the dosing, efficacy and safety of AVP or terlipressin during the study will be recorded. Studies reporting the use of AVP or terlipressin for indications other than hypotension or persistent pulmonary hypotension will be excluded.

\section{Study selection and data extraction}

We will use Covidence as the primary screening and data extraction tool. The titles and abstracts of retrieved studies will be screened by two independent reviewers (ASA and KS) to assess their eligibility. The eligible studies will then be reviewed in duplicate at the full-text level by the same reviewers. A PRISMA flow diagram will be created to illustrate the study selection process. Two reviewers (ASA and KS) will independently conduct data extraction from the full-text studies meeting the inclusion criteria using 
a standardised data extraction form developed in Covidence (table 1). Any disagreements throughout the study selection and data extraction process will be resolved through discussion with a third reviewer (SS-Z).

\section{Assessment of risk of bias and certainty of evidence}

Two independent reviewers (ASA and KS) will conduct a duplicate qualitative assessment of studies using a standardised risk of bias assessment tool appropriate for each study design. The Cochrane risk-of-bias assessment tool for randomised trials (RoB 2), the Newcastle-Ottawa Quality Assessment Scale for cohort and case-control studies and the modified Newcastle-Ottawa scale for cross-sectional studies will be used as appropriate..$^{29} 30$ The quality of descriptive studies and case reports will be evaluated using the Checklist for Analytical Cross-Sectional Studies and Case Reports by the Joanna Briggs Institute. ${ }^{31}$ The same independent reviewers will judge the certainty of the evidence using the Cochrane Grading of Recommendations Assessment, Development, and Evaluation approach. ${ }^{32}$ Any disagreement will be solved through discussion with a third reviewer (SS-Z).

\section{Patient and public involvement}

Patients are not directly involved in the design or conduct of this study. We will plan for public involvement mostly concerned with the dissemination of the results of the review and knowledge translation on the completion of our review, to contribute to wider dissemination of the review to patients and public. We will plan to provide clear explanation of the concept and develop a glossary of research terms specific to our review. We will also provide detailed explanation of the purpose of patient and public involvement and the expectations of their roles. ${ }^{33}$

\section{Outcomes and variables}

Our primary outcomes are (1) improvement in endorgan perfusion defined as an increase in mean, diastolic or systolic blood pressure within 1 hour of the start of treatment, or an increase in urine output, a decrease in the need for inotropes or a reduction in serum lactate within 12 hours of treatment initiation, as defined by the authors in the primary studies, and (2) mortality prior to discharge. Our secondary outcomes are (1) major neurosensory disability defined as moderate to severe motor or cognitive impairment or severe visual or hearing impairment as identified in the primary study; and (2) the occurrence of adverse events defined as peripheral tissue ischaemia, gastrointestinal events (occurrence of perforation, necrotising enterocolitis or gastrointestinal bleed), hepatic events, renal events or hyponatraemia as identified in the primary studies (table 2).

\section{DISCUSSION}

The increased use of AVP and terlipressin in preterm neonates illustrates the need for alternative pharmacotherapeutic options for critically ill neonates. The currently available evidence on the pharmacology of

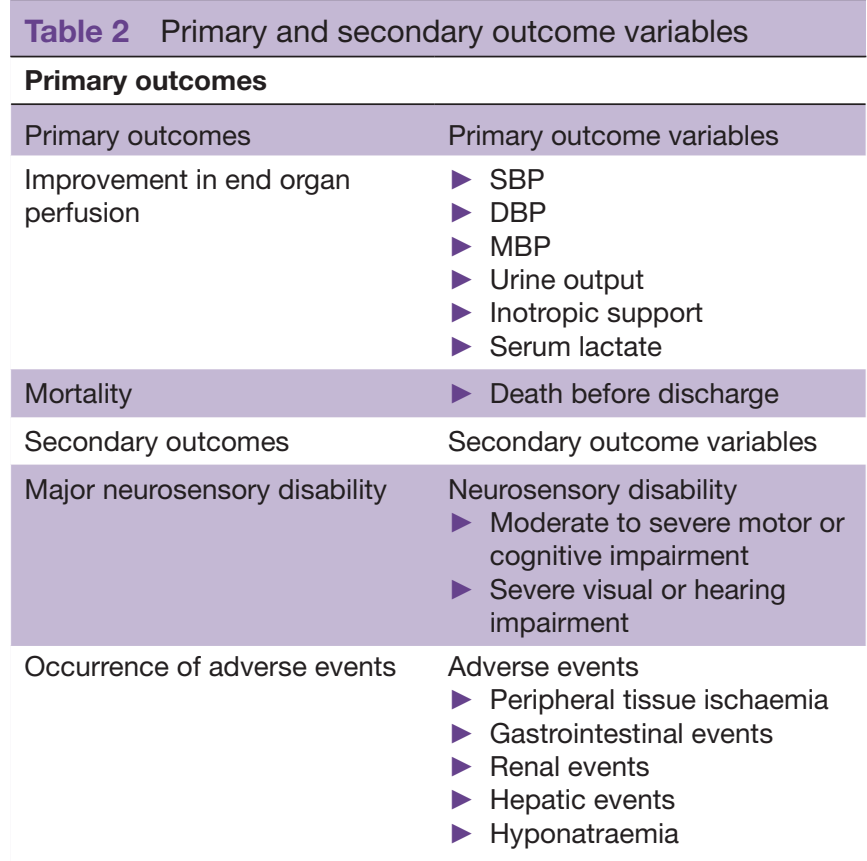

The secondary outcomes will be defined as reported in the primary studies.

DBP, diastolic blood pressure; MBP, mean blood pressure; SBP, systolic blood pressure.

these drugs and the pathophysiology of vasoplegic shock support the biological plausibility for their clinical effectiveness in critically ill preterm neonates. Nevertheless, the available data on the efficacy and safety of AVP and terlipressin in preterm neonates remain limited and in need for a comprehensive review. A systematic review using explicit and reproducible methods helps to produce rigorous and high-quality evidence synthesis and identify evidence gaps that could be the subject of future research. The difficulty in performing clinical trials in preterm neonates limits their evidence-based pharmacological treatment. We aim to complete a high-quality synthesis of the entirety of available data concerning the use of vasopressin and terlipressin among this vulnerable population in order to further the evidence on this important topic.

\section{Author affiliations}

${ }^{1}$ Department of Pediatrics, McMaster University, Hamilton, Ontario, Canada

${ }^{2}$ Faculty of Health Sciences, School of Nursing, McMaster University, Hamilton, Ontario, Canada

${ }^{3}$ Department of Pharmacy, Hamilton, Ontario, Canada

${ }^{4}$ Department of Pediatrics and Pharmacology and Pharmacometric, University Children's Hospital Basel, Basel, Switzerland

${ }^{5}$ Division of Clinical Pharmacology, Children's National Hospital, Washintgon, DC, USA

${ }^{6}$ Intensive Care and Department of Pediatric Surgery, Erasmus MC Sophia Children's Hospital, Rotterdam, Netherlands

${ }^{7}$ Department of Pediatrics, Princess Norah Bint Abdulrahman University, Riyadh, Saudi Arabia

${ }^{8}$ Division of Neonatology, Department of Pediatrics, King Abdullah bin Abdulaziz University Hospital, King Abdullah bin Abdulaziz University Hospital, Riyadh, Saudi Arabia 
Contributors ASA and SS-Z contributed to the conception and design of the protocol. ASA, KS, AR, JVDA and SS-Z contributed to planning the search strategy. $A R, S S-Z$ and JVDA contributed to the critical review of the protocol and review of its scientific content.

Funding The authors have not declared a specific grant for this research from any funding agency in the public, commercial or not-for-profit sectors.

Competing interests I know one of the authors personally and professionally (JVDA). My relationship with this author had no influence on my review.

\section{Patient consent for publication Not required.}

Ethics approval No ethical approval is required as this review will be based on the analysis of publicly available evidence. No personal data of patients will be required. The results of the review will be submitted to a peer-reviewed journal, focusing on paediatrics or neonatology, for publication. We also plan to present results at academic conferences.

Provenance and peer review Not commissioned; externally peer reviewed.

Data availability statement Data are available in a public, open access repository.

Supplemental material This content has been supplied by the author(s). It has not been vetted by BMJ Publishing Group Limited (BMJ) and may not have been peer-reviewed. Any opinions or recommendations discussed are solely those of the author(s) and are not endorsed by BMJ. BMJ disclaims all liability and responsibility arising from any reliance placed on the content. Where the content includes any translated material, BMJ does not warrant the accuracy and reliability of the translations (including but not limited to local regulations, clinical guidelines, terminology, drug names and drug dosages), and is not responsible for any error and/or omissions arising from translation and adaptation or otherwise.

Open access This is an open access article distributed in accordance with the Creative Commons Attribution Non Commercial (CC BY-NC 4.0) license, which permits others to distribute, remix, adapt, build upon this work non-commercially, and license their derivative works on different terms, provided the original work is properly cited, appropriate credit is given, any changes made indicated, and the use is non-commercial. See: http://creativecommons.org/licenses/by-nc/4.0/.

\section{ORCID iDs}

Abdulrahman Salim Alsaadi http://orcid.org/0000-0002-2161-1965

Samira Samiee-Zafarghandy http://orcid.org/0000-0001-8385-0657

\section{REFERENCES}

1 Sharman A, Low J. Vasopressin and its role in critical care. Continuing Education in Anaesthesia Critical Care \& Pain 2008;8:134-7.

2 Henderson KK, Byron KL. Vasopressin-Induced vasoconstriction: two concentration-dependent signaling pathways. J Appl Physiol 2007;102:1402-9.

3 Mavani GP, DeVita MV, Michelis MF. A review of the nonpressor and nonantidiuretic actions of the hormone vasopressin. Front Med 2015;2:19 https://www.frontiersin.org/articles/

4 Holmes CL, Landry DW, Granton JT. Science review: Vasopressin and the cardiovascular system part 1--receptor physiology. Crit Care 2003;7:427.

5 Holmes CL, Landry DW, Granton JT. Science Review: Vasopressin and the cardiovascular system part 2 - clinical physiology. Crit Care 2004;8:15.

6 Bankir L, Bichet DG, Morgenthaler NG. Vasopressin: physiology, assessment and osmosensation. J Intern Med 2017;282:284-97.

7 Lindner KH, Dirks B, Strohmenger HU, et al. Randomised comparison of epinephrine and vasopressin in patients with out-ofhospital ventricular fibrillation. Lancet 1997;349:535-7.

8 Vasudevan A, Lodha R, Kabra SK. Vasopressin infusion in children with catecholamine-resistant septic shock. Acta Paediatr 2005;94:380-3.

9 Filippi L, Poggi C, Serafini L, et al. Terlipressin as rescue treatment of refractory shock in a neonate. Acta Paediatr 2008;97:500-1.

10 Meyer S, Gottschling S, Baghai A, et al. Arginine-Vasopressin in catecholamine-refractory septic versus non-septic shock in extremely low birth weight infants with acute renal injury. Crit Care 2006;10:R71.

11 Australian government, department of health and aging, therapeutic goods administration (2012). Australian public assessment report for terlipressin acetate. Available: https://www.tga.gov.au/sites/default/ files/auspar-terlipressin-acetate-121126.pdf [Accessed 16 Apr 2021]

12 Yildizdas D, Yapicioglu H, Celik U, et al. Terlipressin as a rescue therapy for catecholamine-resistant septic shock in children. Intensive Care Med 2008;34:511-7.

13 Rodríguez-Núñez A, Oulego-Erroz I, Gil-Antón J, et al. Continuous terlipressin infusion as rescue treatment in a case series of children with refractory septic shock. Ann Pharmacother 2010;44:1545-53.

14 Rodríguez-Núñez A, López-Herce J, Gil-Antón J, et al. Rescue treatment with terlipressin in children with refractory septic shock: a clinical study. Crit Care 2006;10:R20.

15 Kam PCA, Williams S, Yoong FFY. Vasopressin and terlipressin: pharmacology and its clinical relevance. Anaesthesia 2004;59:993-1001.

16 Wynn JL, Wong HR. Pathophysiology and treatment of septic shock in neonates. Clin Perinatol 2010;37:439-79.

17 Dünser MW, Mayr AJ, Ulmer H, et al. The effects of vasopressin on systemic hemodynamics in Catecholamine-Resistant septic and Postcardiotomy shock: a retrospective analysis. Anesthesia \& Analgesia 2001;93:7-13.

18 Tsuneyoshi I, Yamada $\mathrm{H}$, Kakihana $\mathrm{Y}$, et al. Hemodynamic and metabolic effects of low-dose vasopressin infusions in vasodilatory septic shock. Crit Care Med 2001;29:487-93.

19 Russell JA, Walley KR, Singer J, et al. Vasopressin versus norepinephrine infusion in patients with septic shock. N Engl J Med 2008;358:877-87.

20 Delmas A, Leone M, Rousseau S, et al. Clinical review: vasopressin and terlipressin in septic shock patients. Crit Care 2005;9:212.

21 Rios DR, Kaiser JR. Vasopressin versus dopamine for treatment of hypotension in extremely low birth weight infants: a randomized, blinded pilot study. J Pediatr 2015;166:850-5.

22 Rios DR, Moffett BS, Kaiser JR. Trends in pharmacotherapy for neonatal hypotension. J Pediatr 2014;165:697-701.

23 Shivanna B, Rios D, Rossano J. Vasopressin and its analogues for the treatment of refractory hypotension in neonates. Cochrane neonatal group, editor. Cochrane Database Syst Rev 2013 http://doi. wiley.com/

24 Mårtensson J, Gordon AC, norepinephrine Tor. Or both in septic shock? Intensive Care Med 2018;44:1964-6.

25 Bidegain M, Greenberg R, Simmons C, et al. Vasopressin for refractory hypotension in extremely low birth weight infants. $J$ Pediatr 2010;157:502-4.

26 Masarwa R, Paret G, Perlman A, et al. Role of vasopressin and terlipressin in refractory shock compared to conventional therapy in the neonatal and pediatric population: a systematic review, metaanalysis, and trial sequential analysis. Crit Care 2017;21:1

27 Shamseer L, Moher D, Clarke M, et al. Preferred reporting items for systematic review and meta-analysis protocols (PRISMA-P) 2015 elaboration and explanation. BMJ 2015;349:97647.

28 McGowan J, Sampson M, Salzwedel DM, et al. PRESS Peer Review of Electronic Search Strategies: 2015 Guideline Statement. J Clin Epidemiol 2016;75:40-6.

29 Sterne JAC, Savović J, Page MJ, et al. Rob 2: a revised tool for assessing risk of bias in randomised trials. BMJ 2019;366:14898.

30 et alWells GA, Shea B, O'Conell D. The Newcastle-Ottawa scale (NOS) for assessing the quality of nonrandomised studies in metaanalyses, 2019. Available: http://www.ohri.ca/programs/clinical_ epidemiology/oxford.asp [Accessed 25 Jan 2021]

31 Moola S, Munn Z, Tufanaru C, et al. Chapter 7: Systematic reviews of etiology and risk. In: Aromataris E, Munn Z, eds. JBI manual for evidence synthesis. JBI, 2020. https://synthesismanual.jbi.global

32 Schünemann H, Brożek J, Guyatt G. Grade Handbook for grading quality of evidence and strength of recommendations. updated October 2013. The grade Working group, 2013. Available: guidelinedevelopment.org/handbook

33 National Institute for Health Research. Public involvement in systematic reviews: supplement to the breifing notes for researchers, 2012. Available: https://www.invo.org.uk/wp-content/uploads/ 2012/10/INVOLVEPublicInvolvementSystematicReviews2012.pdf [Accessed 13 Apr 2021]. 